\title{
EL DOMINIO SOBRE LO COTIDIANO: LA BÚSQUEDA DE LA CALIDAD DE VIDA
}

\author{
Juan Carlos de Pablos \\ Yago Gómez López \\ Nuria Pascual Martínez \\ Universidad de Granada \\ E-mail: jdpablos@goliat.ugr.es
}

\begin{abstract}
RESUMEN
En este trabajo se analiza la materialización de la calidad de vida como uno de los valores dominantes en las sociedades postmaterialistas: qué la produce en la vida cotidiana, qué se hace para lograrla. Partiendo de las distinciones clásicas en el concepto de calidad de vida (elementos objetivos y satisfacción subjetiva; equilibrio y riqueza), se plantean otros componentes en la búsqueda habitual de la calidad de vida. Ésta se hace efectiva a través del consumo, pero lo supera, proporcionando una visión integradora, que abarca desde los elementos de la vida corriente a la propia identidad. Asociada al desarrollo de un proyecto vital, la calidad de vida implica la capacidad para controlar las distintas facetas de la vida. Como contrapartida, supone la continua puesta en juego de los recursos disponibles, en una sucesión continua de tensiones y apuestas que el agente debe resolver para recuperar el sentido de sus relaciones con los entornos social y natural. Se señalan algunas paradojas que impregnan la calidad de vida de un fuerte contenido dialéctico y manifiestan la transformación de las relaciones entre el individuo y la sociedad.
\end{abstract}

\section{INTRODUCCIÓN: CALIDAD DE VIDA Y VIDA COTIDIANA}

Aproximarse al estudio de la vida cotidiana no es una tarea sencilla. Como señala Mike Featherstone (1995: 55), «los comentaristas son raudos en destacar que aventurarse en este campo es explorar un aspecto de la vida cuyos rasgos centrales son, en apariencia, escasamente metódicos, y particularmente resistentes a la categorización racional». Sin embargo, el interés en la vida

\section{Reis}


corriente de la gente corriente es cada vez mayor, pues «la vida cotidiana, con su foco sobre la reproducción y el mantenimiento, las rutinas comunes, la esfera de las mujeres, la receptividad y la sociabilidad, ha ganado ímpetu con la problematización de la legitimidad dominante del mundo de la producción» (Featherstone, 1995: 54). A grandes rasgos, los análisis de Inglehart a partir de diversos estudios de opinión han puesto de relieve algunas transformaciones en la vida cotidiana de quienes viven en las sociedades industriales avanzadas: como consecuencia del incremento en el bienestar material y en la seguridad física, surgen los denominados valores postmaterialistas que, al mismo tiempo que destacan nuevas lealtades supranacionales y preocupaciones ecológicas de carácter general, ponen de relieve con notable énfasis las necesidades individuales de pertenencia, autoestima y realización personal, así como el interés por mejorar en la calidad de vida. Desde luego, con Inglehart, se podría decir que «en toda sociedad industrial avanzada está transformándose lo que la gente espera de la vida» (1991: XXXV).

Trabajos como el de Inglehart, en torno al cambio cultural en las sociedades industriales avanzadas, muestran que los nuevos valores tienen su origen en las grandes transformaciones sociales, pero igualmente que lo que sucede en el entorno inmediato de la gente no es ajeno a su voluntad y a su actuación. El caso español no es diferente. Andrés Orizo (1996), a pesar de constatar, especialmente en los jóvenes, aquello que en la European Value Survey de 1990 se denominó "fragmentación de valores», identifica como valores últimos que sostiene la mayoría de la población el respeto por la vida, la paz, la justicia y la igualdad, la libertad y la expresión, el amor, la solidaridad y la tolerancia. Y lo mismo sucede en el plano individual, donde Orizo encuentra «un perfil del individuo español más contento consigo mismo que hace años, libre y dueño de su destino, que elige y decide por su cuenta en la vida cotidiana y que lo quiere hacer sin intermediaciones» (1996: XXVI).

De acuerdo con Mackay (1997: 7), podemos establecer tres sentidos en el concepto de vida cotidiana:

- En sentido antropológico, el que se refiere a los ciclos secuenciales, marcados por los ritmos que definen las distintas fases de alternancia entre lo excepcional y lo rutinario, lo sagrado y lo profano.

- En sentido político, la vida cotidiana estaría definida negativamente como lo opuesto al «sistema social», regido por las grandes instituciones sociales. Sería el ámbito de lo inmediato y lo pequeño, de la espontaneidad, de los lazos emocionales y la colaboración comunitaria.

- En un tercer sentido, la vida corriente sería el ámbito de la identidad y la expresividad, de la apropiación de los bienes y las representaciones sociales para su reconstrucción en las prácticas verdaderamente significativas para la gente. 
Como vemos, «la práctica cotidiana se organiza en algunas grandes polaridades [...] que pueden captarse a partir de algunas construcciones estructurales» (Maffesoli, 1993: 180). Nuestro punto de partida — tras un estudio empírico de carácter cualitativo sobre la calidad de vida $-{ }^{1}$ se halla en el convencimiento de que la calidad de vida ha venido a convertirse en un auténtico marco de referencia para la vida cotidiana, rico y complejo al mismo tiempo, cuya clarificación puede ser de gran utilidad para la comprensión sociológica del mundo actual. La cuestión se plantea en un momento en que el interés por la calidad de vida como objeto sociológico parece ralentizarse, tras los abundantes estudios de los años setenta y ochenta. Por el contrario, la palabra calidad aparece cada vez con más frecuencia en los contextos de la vida cotidiana, en la publicidad, en las ofertas de nuevos productos y servicios, en los «controles de calidad" cada vez más frecuentes, en las aspiraciones declaradas de las autoridades sanitarias, educativas y asistenciales, etc. En efecto, la cultura de la calidad se abre paso hoy como signo modernizador de nuestro país, desbancando a la trapacería y la chapuza, consideradas durante mucho tiempo señas de identidad de un pueblo aislado y retrasado.

Para la OCDE (1982: 14), la calidad de vida se corresponde con diversas áreas de preocupación social, categorizadas en ocho grandes grupos que representan la estructura del bienestar en sus aspectos fundamentales: salud, educación y adquisición de conocimientos, empleo y calidad de vida en el trabajo, tiempo y ocio, capacidad adquisitiva de bienes y servicios, medio físico, medio social y seguridad personal. Estas áreas se especifican en indicadores sociales concretos para su medida y comparación posterior. Cualquier estudio acerca de la calidad de vida ofrece siempre una lista de los componentes esenciales que sus autores consideran que hay que medir ${ }^{2}$. Sin embargo, nuestro interés se ha dirigido a la configuración de un mapa con las dimensiones básicas que mueven el empeño por lograr la presencia de la calidad en la vida cotidiana, algo difícil de medir, pero fácil de «sentir». De hecho, cuando se pregunta de manera abierta a la gente sobre su calidad de vida, lo primero que se advierte es que consiste en algo diferente para cada cual: los aspectos materiales son los más relevantes para unos, mientras que para otros ocupan el último lugar, desbancados por la satisfacción de estar rodeados por aquellos a quienes se ama; a veces se identifica con la felicidad, y otras con un cierto equilibrio entre logros y aspiraciones; lo que falta todavía por alcanzar está siempre a la vista, dotando a la calidad de vida de una fuerte impronta temporal que trasciende el momento actual.

${ }^{1}$ De Pablos, Pascual y Gómez (1999).

2 Salustiano del Campo (1983: 453) estudia la satisfacción de los españoles relativa a las siguientes dimensiones: trabajo, medio ambiente, vivienda, salud, y con respecto a la vida en general. 


\section{LAS DIMENSIONES OBJETIVA Y SUBJETIVA EN EL CONCEPTO DE CALIDAD DE VIDA}

Como afirma Setién, la calidad de vida es un concepto elusivo y abstracto, complejo y multidimensional, y acerca del cual, especialmente, "la escasez de consenso sobre lo que significa está pesando en los investigadores en este campo, que deben desbrozar caminos, y no acaban de llegar a un cuerpo común de proposiciones» (Setién, 1993: 408). Expresiones como bienestar o nivel de vida suelen ser empleadas como sinónimos de calidad de vida, y en cierto modo lo son: sólo cuando el bienestar material se ha difundido entre amplias capas de la población, ha sido posible el surgimiento del concepto de calidad de vida. La sociedad de consumo ofrece bienes materiales y servicios, puesto que en ella «la mayoría de la población puede satisfacer cumplidamente algo más que sus necesidades elementales» (Castillo, 1987: 12). El mercado y la continua innovación tecnológica se encargan de crear nuevas comodidades, al tiempo que hacen descender las ya asentadas hasta los niveles inferiores. La realidad de la opulencia es un elemento esencial, pues hay que tener cantidad para desear la calidad. Ciertamente, todo esto hace del hecho algo insólito en la historia de la humanidad y los hombres y mujeres han de aprender a gestionar la abundancia y a convivir - si se acepta la expresión- con el consumismo. No es de extrañar, por tanto, que su conceptualización no sea una tarea fácil. He aquí algunos de los conceptos más empleados para expresar la realidad aludida y sus efectos en las personas:

1. El nivel de vida, vinculado de manera evidente a una concepción vertical de la sociedad, según la cual sus miembros se distribuirían de manera ordenada a lo largo de un continuum que hace referencia a elementos más o menos cuantificables, y que ordena a los individuos en capas o estratos.

2. El estilo de vida, caracterizado de manera muy completa por Andrés Orizo (1992: 238), «se forma con la combinación de bienes que uno elige y las actividades que uno hace, todo ello procesado en una configuración más abstracta que viene determinada por los valores que se detentan y la ubicación sociocultural que nos distingue». El estudio de los estilos de vida proporciona una visión de la sociedad multiforme y variada, no vertical, pero su carácter sintético no permite un análisis de la misma más allá de los elementos puramente descriptivos que los conforman ${ }^{3}$.

3. El término género de vida (Ruiz de Olabuénaga, 1998: 35) remite al sistema cultural, y se encuentra a medio camino entre el "estilo de vida" - mediado por la orientación individual de la acción- y el «modo de vida» —referido al sistema normativo institucional-.

4. La expresión tenor de vida ha sido recuperada por Amando de Miguel

3 Para una crítica más amplia del empleo del concepto estilo de vida en la teorización del consumo, véase Campbell (1995). 
(1996: 66) para referirse a la "“constitución u orden firme y estable de una cosa", en este caso, de la vida cotidiana», y estaría compuesto por la posición social (nivel de vida), la situación laboral (modo de vida), la satisfacción vital y el estilo de vida (la manera pautada que se tiene de consumir más allá de la satisfacción de las necesidades perentorias). El interés de este autor es precisamente introducir el elemento subjetivo, para tratar de superar la mera categorización resultante de los estilos de vida. En nuestra opinión, no queda claro de qué manera la satisfacción personal forma parte de los contenidos del tenor de vida sin ser reducida a un elemento descriptivo más, al menos según la definición proporcionada.

5. El concepto de bienestar tiene una amplia tradición en el mundo contemporáneo unido a un tipo particular de organización política, el «Estado del Bienestar», que sería el responsable último de las condiciones de vida de sus miembros. Es un modelo de amplia aceptación social, pues, como señala Morin (1995: 247), "la sociedad burguesa encuentra su ideal en el bienestar, y el movimiento obrero reivindica el bienestar». Desde esta perspectiva sociopolítica, el bienestar incluiría toda una gama de elementos igualmente cuantificables, medibles: bienes materiales, servicios o acceso a los mismos, estudios, salud, derechos civiles y políticos ${ }^{4}$. Por su parte, Nordenfelt (1993: 35) utiliza en inglés dos expresiones para referirse a esta realidad: welfare y wellbeing, distinguiendo matices entre ambas palabras, pues la primera se referiría al bienestar externo, mientras que la segunda sería expresión del bienestar subjetivo de las personas. Desde una perspectiva interpretativa, este segundo elemento, la satisfacción con la vida personal, es especialmente importante, porque su presencia en la vida de los seres humanos condicionará su actuación.

6. La calidad de vida es definida por Setién (1993: 137-138) como «el grado en que una sociedad posibilita la satisfacción de las necesidades (materiales y no materiales) de los miembros que la componen, capacidad que se manifiesta a través de las condiciones objetivas en que se desenvuelve la vida societal y en el sentimiento subjetivo que de la satisfacción de sus deseos, socialmente influidos, y de su existencia poseen los miembros de una sociedad». Este punto de partida está vinculado a una manera de entender y hacer sociología, a partir de grandes encuestas y procedimientos estadísticos que describen parámetros diversos. Es el auge de los indicadores sociales, centrados en el desarrollo de las sociedades, así como en su comparación, ya sea en el tiempo como en el espacio (Galtung, 1972: 253), imprescindibles en el mundo actual. Esta sociología aplicada al conocimiento del desarrollo y la calidad de vida hunde igualmente sus raíces en el «Estado del Bienestar» y tiene su orientación y repercusiones en la figura de los gestores, que precisan de esta información para dirigir sus programas de intervención y política social. De esta manera, la calidad de vida, "como concepto de investigación, se considera un

${ }^{4}$ Determinar en qué medida el interés por la propia calidad de vida se desarrolla correlativamente a la crisis del Estado del Bienestar sería una tarea de gran interés, que excede a este trabajo. 
atributo colectivo, respecto de grupos, categorías de personas, comunidades o sociedades, y no un atributo individual» (Setién, 1993: 114).

La gran limitación de esta perspectiva es que enfatiza la actuación de los agentes macrosociales —administración pública, sistemas sanitario o educativo, empresas - en detrimento de las personas, a las que sólo considera indirectamente, a través de cifras como la cantidad de teléfonos y de vehículos o las ventas de aparatos de aire acondicionado: con respecto a la calidad de su vida, los seres humanos son más bien "pacientes» de lo que sucede a su alrededor, y de las decisiones que toman las instancias sociales. Sin embargo, a la vista de la interiorización de la calidad de vida como un valor por parte de los componentes de la sociedad, se hace necesaria la incorporación de un elemento personal y subjetivo a estas dimensiones para tener una idea adecuada del concepto de calidad de vida, que no puede entenderse sin relación a la satisfacción de quien la posee — como ocurre con el bienestar- . Y, como señalan la mayoría de los autores que tratan combinadamente las dimensiones objetiva y subjetiva de la calidad de vida, es preciso prestar atención al logro de las diversas metas que los sujetos se han propuesto: la «teoría de las discrepancias múltiples» de A. Michalos (1985) pone de relieve cómo la evaluación personal de una situación está mediatizada por las diferencias percibidas al comparar la realidad personal actual con otras realidades, sociales, personales o culturales. Es imposible, por tanto, hablar de calidad de vida sin tener en cuenta la idea de valoración o juicio sobre el nivel de bienestar, aunque toda la concepción de la calidad de vida dependerá de sobre quién recaiga la responsabilidad de emitir esa valoración, y los criterios y variables que se sigan a la hora de medirla.

En resumen: un criterio fundamental para la distinción entre un conjunto de términos tan próximos sería la orientación descriptiva de los primeros, frente al carácter interpretativo que poseerían los últimos —en particular, bienestar y calidad de vida-, que en cierto sentido serían sinónimos, al caracterizarse por la posesión de un determinado grado de nivel de vida y por la satisfacción de quienes lo disfrutan.

\section{EQUILIBRIO EN LAS ASPIRACIONES Y RIQUEZA EN LA CALIDAD DE VIDA}

La distinción anterior establece dos ejes o dimensiones (bienestar objetivo y satisfacción subjetiva) imprescindibles para la conceptualización de la calidad de vida, pero no resuelve el problema de la causa de la satisfacción personal, pues se da la paradoja de cómo personas con un menor nivel de vida material pueden sentirse más satisfechas que otras en mejores condiciones, o lo atribuye a razones de índole cultural. De ahí que Nordenfelt se plantee el siguiente dilema: o bien bienestar objetivo y bienestar subjetivo son dos cosas distintas, o bien, para medir una cosa, el bienestar subjetivo, precisamos de dos tipos de 
datos, objetivos y subjetivos. Y Nordenfelt (1993: 15) se inclina por la segunda opción, de manera que la cuestión podría plantearse entonces como "¿cuáles son aquellos estados y aspectos externos en la vida de un ser humano que tienen una conexión tal con el bienestar subjetivo de la persona que podamos utilizarlos en un contexto científico como indicadores de bienestar?». Para responder a esta cuestión, Nordenfelt introduce una nueva dualidad en la calidad de vida: una cosa sería el equilibrio entre los deseos y la realidad personal que cada uno tenga, que es siempre subjetivo, y otra la riqueza objetiva que pueda hallarse en una determinada situación, personal o colectivamente. Nordenfelt plantea la diferencia que existe entre una persona que esté contenta con los logros y las aspiraciones de su vida —más o menos organizados alrededor de una escala de prioridades - y otra que posea una vida llena de elementos que la enriquecen: la "riqueza» de una vida es una dimensión que no se refiere a la relación entre aspiraciones y aspiraciones satisfechas, sino al número y la naturaleza de esos deseos y condiciones vitales. Ésta sería la dimensión más importante de la calidad de vida, por cuanto es expresión objetiva de la causa del bienestar de la persona. Lógicamente, no se reduce a los bienes materiales, que ocupan el lugar inferior en la escala, dejando hueco a los de orden más elevado, intelectual y espiritual. Paradójicamente, el límite superior de la «riqueza» de la calidad de vida depende de las características individuales, de la diferente capacidad de cada uno para ser feliz.

Esta distinción entre equilibrio y riqueza en la calidad de vida, a pesar de perfeccionar considerablemente el concepto, no termina de resolver el problema de la causa de la satisfacción, sino que más bien lo complica, al señalar para ella dos fuentes de índole diferente, que están presentes en el estado actual de quien posee la calidad de vida. Además, las dos tienen un componente subjetivo y otro objetivo:

1. La riqueza de la calidad de vida se asemeja a las medidas objetivas del bienestar y el nivel de vida. Y a partir de un determinado nivel, en contextos similares, no es fácil establecer qué proporciona más riqueza: ¿Una segunda residencia en el mar o en la montaña? ¿Es mejor tener un barco que una avioneta? ¿Enriquece más un viaje por América o por Asia? Si dejamos a un lado las diferencias culturales, vuelve a ser cuestión de preferencias personales, del goce o satisfacción que le produzca a cada uno.

2. El estado de satisfacción en que se encuentra el agente de la calidad de vida no es sólo una cuestión interna, sino también un hecho objetivo, pudiendo diferenciarse quienes se hallan en esa situación de equilibrio de quienes no lo están. ¿Qué sucede cuando el equilibrio se rompe en el momento en que el individuo trata de incrementar la riqueza de su calidad de vida? 


\section{LA CALIDAD DE VIDA COMO ESTADO Y COMO PROCESO}

La calidad de vida no es, por tanto, la satisfacción individual con la vida, ni el resultado de la suma numérica exacta de unos determinados indicadores. Se parece más bien a la resultante de la interacción de un amplio conjunto de aspectos, donde tienen lugar sumas, pero también restas, y donde el valor de los sumandos y restandos no es siempre el mismo. Tratar de identificar estos elementos, así como la forma en que se articulan entre sí para lograr el resultado final, nos conduce a dos interrogantes: ¿En qué se piensa cuando se piensa en calidad de vida? ¿Cómo se comportan los seres humanos cuando intentan conseguirla o mejorarla? De esta manera, surge una nueva clasificación - la calidad de vida como estado y la calidad de vida como proceso- que contrasta con las dimensiones anteriores, pero que permite destacar aspectos relevantes que de otra forma quedarían ocultos 5

La consideración de la calidad de vida como el estado de cosas que se disfruta en un determinado momento permite establecer de qué manera se relacionan diversos elementos entre sí para proporcionar la calidad y la satisfacción. El agente de la calidad de vida se halla siempre en una situación concreta, disponiendo de unos recursos que orienta hacia determinadas actividades, que proporcionan la calidad:

1. La situación define el contexto en el que se ha de mover el agente, e incluye tanto el medio natural como el entorno sociopolítico y cultural. Establece los límites en los que se desarrolla la acción orientada a la calidad, pero lo hace en un sentido ambivalente, a la manera como los modernos Estados burocráticos permiten el surgimiento de la democracia y la libertad personal, restringiendo y potenciando al mismo tiempo la capacidad de actuación en una u otra dirección: la calidad de vida está estrechamente ligada a las relaciones con el entorno.

2. La sociedad capitalista ha dotado a las clases medias de abundantes recursos para poner en juego. Desde la perspectiva de la calidad, cualquier cosa que pueda utilizarse con carácter instrumental es recurso: medios materiales -dinero, casa, coches-, capital cultural, posición y relaciones sociales. Incluso el carácter, las capacidades, las habilidades sociales de cada uno. Los recursos son la base del nivel de vida, que no puede lograrse sin ellos.

3. En las actividades que se llevan a cabo se halla la satisfacción intrínseca de la calidad de vida: el goce y la frecuencia, asociados a la posibilidad de adecuación individual a través de la elección. Además, muchas actividades instrumentales — trabajar, cocinar, limpiar, etc.— se han librado de sus aspectos más onerosos. El caso extremo son las compras, que han acabado por convertirse en una actividad finalística más, fuente de placer.

5 Ya Setién había distinguido entre la calidad de vida como proceso y como producto (1993: 114), pero el empleo de técnicas cuantitativas limita la calidad de vida al producto, aunque se reconozca teóricamente la influencia del proceso. 
Es fácil advertir la relación entre estos elementos, pero - a nuestro juicio- es imposible ponderar o establecer categorías exactas para determinar su influencia en la calidad de vida de las personas, sobre todo porque aquello que en un momento actúa como limitación — los hijos son el caso paradigmático por excelencia, fuente de gastos y quebraderos de cabeza- puede acabar por convertirse en la mayor fuente de satisfacción. De ahí que sea nuevamente el papel que el propio agente juega con relación a la calidad lo que determine efectivamente su posesión o su falta. Y esto, de manera dinámica, vinculada al proceso por el que se busca y consigue la calidad de vida:

1. Para el agente de la calidad de vida, ésta se convierte en un continuo discernir entre el amplio abanico de posibilidades que la sociedad ofrece, a través del desarrollo de continuas tensiones que es preciso resolver de una u otra forma.

2. La solución tendrá siempre el carácter de apuesta, pues la satisfacción no está garantizada de antemano: no es posible conocer con anticipación si finalmente la opción escogida será o no del agrado del individuo. Sin embargo, toda apuesta origina la efectiva orientación de los recursos del agente en una dirección determinada. En cierto modo, podríamos comparar el juego de la calidad de vida con un juego de estrategia, coherente con el empleo de recursos limitados en orden a la obtención de determinados objetivos ${ }^{6}$. La naturaleza de este proceso se halla en el intercambio de recursos entre la sociedad y los individuos, a través de la mercantilización de los bienes en un mundo interdependiente.

3. El proceso culmina con la comprensión por parte de los individuos del sentido de su propia actuación, donde nos parece que se halla el punto más importante. A continuación veremos algunas de sus implicaciones.

\section{RACIONALIDAD DE LA CALIDAD DE VIDA: LA PARTE Y EL TODO}

Por definición, la calidad de vida atiende a la totalidad de la vida, no sólo a una parte. Pero son precisamente las distintas actividades parciales las que proporcionan bienestar, al poner en juego los recursos disponibles según determinados criterios. Por ejemplo, la dimensión temporal está dominada por la posibilidad de obtener inmediatamente la gratificación o posponerla para más tarde. También podemos considerar la satisfacción que puede procurar un determinado bien, a costa de las demás parcelas de la vida. En general, podrían establecerse dos grandes categorías de comportamientos con relación a la calidad:

${ }^{6}$ Utilizamos la expresión «juego de estrategia» como metáfora, no como identificación entre ambas realidades. Alonso y Callejo (1994: 130) muestran cómo ninguno de los juegos desarrollados en la bibliografía del individualismo metodológico se ha podido aplicar al consumo. No obstante, la calidad de vida se rige por otros parámetros, pues es globalizadora de recursos y actividades, mientras que el consumo es inmediato y particularista. 
1. Recursos para Actividades $(\mathrm{R} \rightarrow \mathrm{A})$ : La calidad — goce, elección- se alcanza cuando una parte se destaca en el todo de la vida, proporcionando satisfacciones que no se disfrutan de manera frecuente ni continuada. No quiere decirse que no haya pequeños o grandes placeres en la vida, sino que el carácter instrumental — la supervivencia- domina las actividades que se realizan: el sentido del ocio es el descanso, reponer fuerzas para continuar trabajan$\mathrm{do}^{7}$. Para este procedimiento, propio de las sociedades tradicionales, en el que algunas actividades destacan especialmente en el todo de la vida, hemos escogido la denominación de modo materialista de la calidad de vida, siguiendo la tradición iniciada por Inglehart: al depender de los recursos, el énfasis se pone en su consecución y disponibilidad, pues sin ellos todo lo demás está vetado.

2. Actividades frente a Actividades $(\mathrm{A} \leftrightarrow \mathrm{A})$ : El modo propio de una sociedad de la abundancia se caracterizaría por la presencia de numerosas fuentes de satisfacción al mismo tiempo. Los recursos no son infinitos, pero son suficientes para que el sello de la calidad esté presente en la mayoría de las acciones de la vida cotidiana, incluyendo la vida laboral. Las tensiones siguen presentes, pero ahora tienen lugar de manera que una parte resta satisfacción al bienestar conjunto que proporcionan los demás elementos en la vida del agente. El trabajo puede considerarse desde varias perspectivas: como una actividad que se desea por sí misma, capaz de proporcionar determinadas gratificaciones de tipo personal o social que ninguna otra actividad puede lograr ${ }^{8}$, o bien como un elemento que roba tiempo de ocio o de dedicación a la familia. Existen otras muchas tensiones: comida saludable frente a los placeres de la buena mesa, elección de actividades de ocio y esparcimiento, el «maravilloso universo" de las compras, la vida en el campo o en la ciudad, el tiempo de trabajo frente al tiempo de vacaciones y, para el caso de las mujeres, la tensión característica entre trabajo extradoméstico, cuidado de los hijos y tareas del hogar. La visión del que hemos denominado modo postmaterialista de la calidad de vida se caracteriza, paradójicamente, en medio de la abundancia, no por lo que se tiene, sino por lo que todavía falta, identificando con relativa facilidad no las fuentes de placer, como en el modo materialista, sino las de frustración e insatisfacción.

Lo primero entonces es recuperar el balance perdido. Como la calidad de vida trata sobre las relaciones entre la parte y el todo, es preciso integrar la nueva tensión de alguna manera: será preciso elegir, renunciar a alguna actividad entre las que se llevan a cabo, dejar de practicar alguna para comenzar otra nueva que se muestra como más apetecible o conveniente. Pero muchas veces no es posible renunciar al estado de cosas vigente, bien porque cuesta decidir,

Una excelente discusión acerca del sentido social del ocio puede encontrarse en Ruiz de Olabuénaga (1994), donde se rebate el planteamiento instrumental del ocio, caracterizándolo por sus aspectos emocionales (Elias y Dunning, 1992). Sin embargo, si atendemos a la centralidad del ocio o del trabajo en la vida cotidiana de las personas, las cosas cambian (Rojek, 1995).

8 Por ejemplo, Pérez Adán (1991: 1903). 
bien porque la resolución de un dilema tarde en resolverse (préstamos e hipotecas), bien porque en la práctica es imposible su desaparición, como el caso de quien desea un trabajo más flexible o satisfactorio, o el de muchas amas de casa que desearían trabajar y no encuentran un empleo conveniente. A veces, la capacidad de adaptación puede facilitar mucho las cosas, como cualquier otro recurso personal, pero la verdadera solución llega mediante la reflexión. No se trata ya solamente del hecho de que en las sociedades postindustriales se favorezca el desarrollo de habilidades intelectuales, sino que los agentes son impulsados a ellas, como consecuencia de la necesidad de recuperar el sentido, que pasa por la conciencia de la propia situación, posibilidades y limitaciones ${ }^{9}$. Las principales implicaciones son dos:

1. El proceso de toma de decisiones es continuo, aunque no quiere decir que éstas se adopten siempre con arreglo a criterios constantes o de racionalidad económica. Como señala Jameson (1996: 209), se está difundiendo un nuevo concepto de «decisiones "racionales" en el nuevo y más amplio sentido de aquello que cualquier otro ser humano podría comprender (en el sentido de Dilthey o de Rousseau, todo aquello con lo que cualquier otro ser humano podría "simpatizar")».

2. La calidad de vida tiende de suyo a una cierta racionalidad, por el hecho básico de atender a la totalidad: cuidar la salud, regular los placeres, equilibrio de los recursos, etc. Featherstone (1991: 81) utiliza la expresión «calculado hedonismo» para referirse a las «técnicas que permiten al yo el desarrollo de sensibilidades que posibilitan disfrutar el balanceo entre los extremos [...] de manera que los placeres de la inmersión y el distanciamiento pueden ser disfrutados a la vez». Por eso, lo relevante es la presencia de tensiones: la clave no es gastar ahora o luego, en una cosa u otra, sino la presión incesante hacia la mejora de la calidad, que continuamente desestabiliza el equilibrio alcanzado y mueve a la actividad.

\section{PRESIÓN SOCIAL E IDENTIDAD PERSONAL}

En las sociedades industriales avanzadas, muchas personas desarrollan un proyecto personal para su vida, que les permita formular objetivos y medios para alcanzarlos ${ }^{10}$. Existen evidencias empíricas de este tipo de planes, en los

9 Quizá no haya satisfacción mayor ni mejor fuente de sentido que la de verse dueño del propio destino y serlo, en la medida de lo posible. Sirva como ejemplo esta afirmación de un joven profesional universitario (24 años, casado, vivienda individual adosada en la periferia de la ciudad): «Tenemos una independencia económica y decidimos lo que queremos hacer. Lo haremos bien o mal, pero lo estamos decidiendo nosotros. No nos lo está imponiendo nadie» (De Pablos, Pascual y Gómez, 1999: 70).

${ }^{10} \mathrm{Su}$ presencia nos parece un elemento definitivo a la hora de conceptuar los elementos que componen la calidad de vida, y de establecer los rasgos del «agente» que la busca activamente. 
que se integran de manera jerárquica diversos objetivos, organizados según escalas de valores que rigen los comportamientos concretos. Esto no quiere decir que esté igualmente claro para todo el mundo el sentido de la vida, ni los últimos fines que se han de buscar, ligados al problema de la felicidad y a otras cuestiones éticas. En cierto modo, el proyecto personal, que no tiene por qué ser algo perfilado y ni siquiera consciente, no tendría por qué ser más que un mínimo de comprensión de la propia vida y del entorno, y una jerarquización en las pretensiones del individuo ${ }^{11}$.

Desde luego, el proyecto vital no es un concepto que haya sido empleado con anterioridad por la sociología, pero existen múltiples referencias en el conjunto de conocimientos que la rodean. Desde la psicología social, Maslow (1982) había situado la realización personal en la cumbre de su jerarquía de necesidades. En un plano filosófico, José Antonio Marina ha puesto de relieve la continua búsqueda de la felicidad y de la perfección que poseen los seres humanos, integradas en la capacidad de generar proyectos y expandir la realidad, de incrementar continuamente el número de posibilidades a las que el ser humano es capaz de enfrentarse, en una continua búsqueda de «lo mejor» (Marina, 1995: 155). Desde una perspectiva que atiende a las transformaciones que nos rodean, Handy (1996: 88) señala las nuevas posibilidades que se abren para las personas en el mundo presente, en la necesidad de poseerlo y dominarlo, antes de que él se imponga: «El auténtico "nosotros" está, irónicamente, no en el núcleo, sino en el todo, si pensamos en el núcleo como las cosas necesarias para la vida, ese curriculum vitae que tan asiduamente elaboramos en nuestros primeros años. Está en nuestras manos, y sólo en nuestras manos, llenar el espacio antes de morir.»

Éste es el contexto social que Giddens (1995: 26) caracteriza como «un orden post-tradicional en el que a la cuestión “¡cómo he de vivir?” hay que responder con decisiones tomadas cada día sobre cómo comportarse, qué vestir, qué comer - y muchas otras cosas—; además, tal cuestión se ha de interpretar en el despliegue de la identidad del yo en el tiempo». El proyecto personal es, por tanto, una forma - entre otras posibles- de organizar la toma de decisiones que exige la calidad de vida. En cierto modo, la primera tensión presente en el contexto de la calidad de vida es la que existe entre el individuo y la sociedad: la necesidad de autoafirmación, el desarrollo de la identidad, las condiciones para la propia determinación de la vida, son el primer requisito de la calidad, visible en los más jóvenes, más próximas a los ideales postmaterialistas ${ }^{12}$. La construcción de la propia identidad se ha convertido en un auténtico reto, una auténtica forma de "creatividad humana» (Squicciarino, 1990: 185). Pero cualquier objetivo o meta personal está marcado por la pre-

11 Sobre el concepto de pretensiones individuales y sus implicaciones en la vida social, véase Luhmann (1995).

12 Para el estudio de las condiciones sociales que han hecho posible el desarrollo del individuo y la individualidad pueden verse, entre otros, Durkheim (1987) y Luhmann (1995). Para una visión crítica de las paradojas del individualismo, Béjar (1995) y Camps (1993). 
sencia del mercado, de manera que las personas «crean un sentido de quiénes son a través de lo que consumen" (Bocock, 1995: 102).

¿Se ha convertido el consumo individual, entendido como el medio principal para obtener calidad de vida, en el sustituto de la democracia en la sociedad de masas? ¿O es simplemente su realización definitiva? Parece como si, a medida que la sociedad crece cuantitativamente y se complejiza cualitativamente, se alejase del añorado paradigma de la participación ciudadana en la toma de decisiones políticas. La libertad es uno de los pilares básicos de los sistemas democráticos, pero frecuentemente ésta queda reducida a la posibilidad fáctica de elegir o votar a un partido u otro. El consumo, donde la gama de posibilidades se estira prácticamente sin límite, supera ampliamente la oferta de elección política. Podríamos pensar que la progresiva apolitización de las nuevas generaciones tiene su correlato en el repliegue hacia el consumo individual y la búsqueda de calidad de vida. Para Featherstone (1991: 83), "la cultura del consumidor contemporáneo connota individualismo, personalidad y conciencia del propio estilo. El propio cuerpo, la indumentaria, la forma de hablar, las actividades de ocio, las preferencias en comida, bebida, casa, coche, elección de vacaciones, etc., deben verse como signos de individualismo en el gusto y sentido del estilo del propietario/consumidor».

Sin embargo, aunque la calidad de vida se haga efectiva a través del consumo, son dos realidades distintas. Desde el punto de vista de quienes están convencidos de que el consumismo voraz y acrítico (por ejemplo, Gutiérrez Brito, 1995) domina el comportamiento de la gente, la calidad de vida sería algo inalcanzable, siempre escondida detrás de las fútiles y efímeras satisfacciones que proporcionan los caducos bienes de consumo. Pero, como señala Savater (1997: 24), "la gente, para saber lo que le gusta o lo que no le gusta, tiene que tener a mano la potencialidad de disfrutarlo. [...] Lo importante es tener las cosas, pero que éstas no terminen adueñándose de la persona. [...] Para nuestra vida normal hay dos impedimentos: uno, carecer de cosas; el otro, tener tantas que uno no se pueda mover». La idea de la calidad de vida propone la existencia de una cierta unidad y continuidad de criterio en las personas, pues lo importante de cada cosa es su «capacidad de crear bienestar, y es el bienestar final lo que constituye el criterio último para su importancia» (Nordenfelt, 1993: 41).

Hallaríamos otra diferencia entre consumo y calidad de vida en la visión que se tiene del primero como irracional, impulsiva e instintiva ${ }^{13}$, que contrasta con la segunda, reflexiva y valorativa. Mientras las actividades de consumo tienen carácter puntual, el interés por la calidad se presenta como totalizador en sus exigencias: el dinero y los objetos dejan de ser finalidades en sí mismos,

13 En nuestra opinión, los consumidores no son tan fácilmente manipulables como a veces se tiende a pensar. Si así fuera, no habría fracasos en las promociones. Las campañas de ventas y publicidad se dirigen a un público cada vez mejor informado y exigente, mientras los expertos en márketing necesitan continuamente nuevas ideas (Rodríguez, 1997). 
para ser instrumentos vinculados a una meta más o menos general. Es posible que, en las relaciones entre consumo y calidad de vida, lo racional no esté reñido con lo irracional, coexistiendo "pacíficamente»: lo racional puede ser dejar un espacio para cierto consumo compulsivo, en una o varias áreas - por ejemplo, ropa, discos, libros, cosméticos, dulces, etc._-, controlando el gasto en el resto. Si esto no se logra, puede surgir entonces la patología, el shopaholism, de carácter socialmente limitado, como todas las patologías ${ }^{14}$. Algo similar sucede con fumar, beber, conducir con exceso de velocidad o practicar deportes de riesgo.

Independientemente de los patrones de consumo que se sigan, los estudios sobre cultura del consumidor (Bocock, 1995) muestran a éste cada vez más exigente, más consciente y empeñado en la calidad de lo que adquiere, poseedor de una mayor preocupación por las consecuencias de sus actos, acorde con aquello que se llamó, en los primeros grandes estudios sobre la sociedad de consumo, el "poder del consumidor» (Katona, 1968: 8). Y, desde luego, los estudios de mercado carecerían de sentido si no fuera preciso salvar continuamente una distancia entre el sistema de producción y la vida real de los consumidores, cada vez más adaptados a los nuevos valores postmaterialistas. Así, pues, la calidad de vida se hace efectiva a través de la sociedad de consumo, pero la supera, al introducir criterios globales de satisfacción y regularlos a través del proyecto vital.

\section{ENTRE LO NECESARIO Y LO POSIBLE}

A estas alturas, podemos ofrecer una definición de calidad de vida, que entendemos como la disponibilidad, por parte del agente, de recursos y actividades para sacar adelante un programa de vida más allá de la mera razón de supervivencia, es decir, del sentido instrumental del trabajo y las actividades cotidianas como medio de subsistencia. En otras palabras, se tiene calidad cuando se está en condiciones de controlar la propia vida, así como el medio en que ésta se desenvuelve, el conjunto de circunstancias que rodean la propia existencia. No sólo cuando se posee mucho nivel de vida material, ni tampoco cuando se está satisfecho con lo que se tiene, aunque sea «muy poco» y se piense que nada más es necesario.

Controlar la propia vida, para quienes carecen efectivamente de los medios para que nadie o muy poco escape a ese control, puede lograrse haciendo de la necesidad virtud, aceptando la situación de manera que lo dado de antemano acabe por convertirse en lo deseado, asumiendo lo establecido como algo que tiene que ser así. Pero también cabe el esfuerzo por mejorar las condiciones de vida, integrando la riqueza existente en la vida personal, a través de un juego de intercambios simbólicos y materiales, vinculado a las posibilidades reales de

14 Sobre las expresiones shopaholism y shopaholics, cfr. Revista de El Mundo, 7-I-1996. 
cada uno. Ambas situaciones se dan en la vida cotidiana; lo cual, aplicado a la búsqueda de la calidad de vida sugeriría nuevos puntos de vista:

- La dialéctica entre la asunción de lo necesario, de lo que «tiene que ser así» a través de la reflexión personal y de la búsqueda del sentido, y la apertura, a través de la acción orientada a la calidad para hacer real lo $p_{0 s i b l e}{ }^{15}$. En la vida cotidiana, avanza poco a poco, optimizando aquellas parcelas que pueden modificarse, mientras racionaliza las que no pueden cambiarse.

- Además de la relación existente entre recursos y actividades, es central la vinculación con el contexto, porque algunas situaciones, incluyendo los medios natural y sociocultural, son más manejables que otras.

Encontramos así otra clave en el esfuerzo que hay que realizar para alcanzar determinados niveles de riqueza y satisfacción, procurando al mismo tiempo mantener o incrementar los recursos disponibles. La realidad es que sólo se puede llegar a poseer un control limitado de la propia existencia: un cierto nivel de vida objetivo, pero no el que se quisiera verdaderamente poseer; un cierto equilibrio entre las aspiraciones y los logros, que no satisface totalmente; una riqueza vital y una satisfacción que siempre pueden ser superadas. Estas dimensiones son condiciones necesarias para la calidad de vida, pero ninguna responde totalmente al concepto — desde una perspectiva sociológica- porque la clave está en la capacidad para controlar la propia vida, para decidir sobre ella, para escoger entre los diversos caminos posibles, en lo material y en lo simbólico, en lo personal y en lo social. De esta manera, además, se puede explicar la paradoja de cómo quienes viven en peores condiciones de vida material y de acceso a bienes y servicios de gran relevancia pueden sentirse más satisfechos que quienes poseen un mayor nivel material de vida y, por tanto, condiciones más favorables para la calidad de vida. Llegamos así a desenvolvernos en un ámbito estructural de la calidad de vida: ya no es sólo cuestión personal, pero tampoco exclusivamente cultural.

El resultado es una primera paradoja acerca de la calidad de vida: intentar alcanzarla o incrementarla supone renunciar al bienestar material máximo que podría llegar a conseguirse, a favor de una conciencia de la propia situación, de la comprensión de las propias necesidades y posibilidades, de la optimización del esfuerzo y de los recursos, es decir, del desarrollo de un proyecto personal que implica tanto la consecución de algunas metas como la renuncia a otras.

15 Resulta así una aplicación en el ámbito de lo cotidiano del principio básico de las modernas teorías del cambio social, que resaltan el papel de la agencia humana por encima de cualquier tipo de determinismo o de causalidad extrasocietaria: la historia es obra humana, aunque no se desarrolla en las condiciones que nos gustaría, sino con limitaciones dadas y según mecanismos no del todo controlables (Sztompka, 1995). 


\section{DEL SUJETO AL AGENTE DE LA CALIDAD DE VIDA}

Podríamos denominar sujeto de la calidad de vida a la persona que disfruta de determinado grado de bienestar y satisfacción personal. Todo el mundo es sujeto de un determinado nivel de vida, aunque no todo el mundo es sujeto de la misma calidad de vida, según las categorías objetivas establecidas. Ahora bien, no es lo mismo la situación de quienes conscientemente buscan mejorar su calidad de vida que la de quienes no se lo han planteado. Por eso, denominamos agente de la calidad de vida a quien deliberadamente se implica en su búsqueda, aunque quizá no sea fácil determinar cuándo una persona se convierte en tal. Como mínimo, implicaría un cierto grado de reflexión sobre la propia situación, así como algunas decisiones efectivas referidas a su bienestar.

La cuestión tampoco es baladí si consideramos la dimensión grupal de la calidad de vida, pues lo que podríamos denominar «unidad básica de calidad de vida» no es exclusivamente el individuo, sino la familia, para quien se busca la calidad en su conjunto como extensión del propio "yo» del agente. Desde luego, se da una considerable identificación entre los miembros de la unidad familiar: verlos contentos supone una de las mayores fuentes de satisfacción. $\mathrm{Y}$ es que las decisiones con respecto a la calidad no se toman exclusivamente en función del interés personal de quien ha de poner en juego los recursos, sino de quienes conviven en el hogar. Y aplicando el mismo criterio de interés por la calidad de vida de los que conviven con el agente, es preciso tener en cuenta no sólo las «nuevas formas familiares» (Iglesias de Ussel, 1994), sino también la convivencia entre personas sin lazos de parentesco: el hogar sería una cierta unidad espontánea de la calidad de vida ${ }^{16}$. De esta manera, la calidad resalta las condiciones sociales por encima de las materiales: la situación de un estudiante en un piso, con "colegas», puede llegar a ser un hogar, pero también todo lo contrario; más bien se aproxima a la idea de «comunidad emocional» de Maffesoli (1990: 43), que permite la integración en un conjunto, que "puede constar de una pluralidad de elementos: siempre hay un ambiente específico que los torna solidarios entre sí».

La calidad de vida define, por tanto, el entorno habitual o inmediato en el que se desarrolla la vida de las personas en las sociedades avanzadas, desde los más pequeños detalles a lo que posee mayor relevancia. Todo invita a la consideración de este ámbito personal como una especie de estuche o burbuja, donde se llevan a cabo gran parte de las actividades más valoradas, que de alguna manera conforman los límites de la vida de las personas. Sin embargo, éstos son cada vez más cambiantes, flexibles y en continua expansión, de manera que uno de los rasgos más llamativos es la capacidad que tienen los hombres y mujeres de hoy para influir sobre su alrededor, con posibilidades de interven-

${ }^{16}$ Hogar se define como la «unidad económica y social constituida por un conjunto de individuos que conviven habitualmente bajo el mismo techo y ocupan la misma vivienda» (Centre d'Estudis Demografics, 1990: 5). 
ción impensables hace sólo unas décadas. Esto ha de cambiar necesariamente la manera que tiene el ser humano de relacionarse con el entorno, descubriéndole que puede hacer algo a su favor o en su contra.

Por eso, encontramos también paradójica la calidad de vida cuando nos acercamos con detalle a esta burbuja que parece a simple vista, y observamos ciertos rasgos universalistas en el estuche: la calidad se busca no sólo para uno mismo, sino para toda la familia, primero, y para el conjunto de personas con el que uno se relaciona. Además, la calidad de vida no puede limitarse a un entorno reducido, puesto que la de los otros afecta a la propia: la calidad de vida no es «mi bienestar», puesto que afecta al aire, al agua, al medio físico y al medio social, y se hace efectiva en el trato interpersonal. Por naturaleza, la calidad de vida no puede ser el manto bajo el cual se aísle el individuo del resto del mundo. Surge en las actividades cotidianas un sentido universalista, concretado en la preservación de la naturaleza y en la calidad de vida de los demás, absolutamente nuevo.

Por último, y no menos importante, la búsqueda de la calidad de vida se comporta como un mecanismo por el cual se da reconocimiento al derecho de cada uno a intentar alcanzarla en las actividades que prefiera y a través de los medios que considere oportunos: el contexto de la calidad de vida es la sociedad civil, entendida en su concepto más amplio, es decir, no por su oposición al Estado, sino como forma de organización social, propia de «sociedades civilizadas», poseedoras de una cultura cívica (Pérez Díaz, 1993: 70) ${ }^{17}$. Es cierto que la sociedad es individualista, pero si lo interpretamos como forma de organización social, quizá tengamos que repensar el sentido de las conocidas palabras de Tocqueville (1989: 89): «El individualismo es un sentimiento reflexivo y apacible que induce a cada ciudadano a aislarse de la masa de sus semejantes y a mantenerse aparte con su familia y sus amigos; de suerte que después de formar una pequeña sociedad para su uso particular, abandona a sí misma a la grande.» Es la segunda paradoja de la calidad de vida: ésta se hace efectiva a través de la cultura del consumo, pero lo trasciende; parece un estuche en el que se encierran los individuos, pero en realidad es una burbuja global que los vuelve conscientes de sus relaciones con el resto del mundo, desde el momento en que el agente advierte a los demás como sujetos de calidad de vida. Por otra parte, la calidad de vida es tan sólo una puerta abierta: en un contexto postmaterialista de la vida, la sociedad exige un mínimo de comportamiento ético, pero no propone elevadas metas con carácter universal.

17 Fukuyama (1992) recoge dos tradiciones filosóficas presentes en los fundamentos de la sociedad civil: la liberal anglo-escocesa, basada en los criterios de propiedad y libertad; y la hegeliana, apoyada en la superación del conflicto entre el señor y el esclavo y, por tanto, en el reconocimiento mutuo. 


\section{LIBERTAD Y CONTROL EN LA CALIDAD DE VIDA}

Por último, consideraremos otro tema que, igual que el anterior, trata de las conexiones entre los aspectos macro y microsociológicos del mundo actual $^{18}$. El carácter procesual y cíclico que caracteriza la búsqueda de la calidad de vida hace que ésta se haya convertido en un auténtico motor de la actividad humana en las sociedades avanzadas. El empeño por mejorar la situación personal y familiar ha pasado a convertirse en el leitmotiv de los hombres y mujeres del tercer milenio, ya que posee una fuerza capaz de dotar de sentido —individual y colectivo - al comportamiento humano. La posesión de determinados niveles de calidad no puede dejar de tener sus efectos sobre los seres humanos. Además, o quizá precisamente por eso, la calidad de vida se ha tornado un bien jurídico de suma importancia, algo que debe ser salvaguardado ante posibles amenazas externas y un objetivo político de carácter programático de primer orden: es patente la dificultad para rebatir cualquier discurso centrado en la calidad de vida, hecho que apunta hacia la conexión entre ésta y la ideología, y a un proceso de "sustitución funcional».

La búsqueda de la calidad de vida exige en el agente un proceso de aprendizaje de las reglas del juego, para dominarlas y, por tanto, optimizar el ritmo y el beneficio que se obtiene. Este «saber hacer» conlleva multitud de implicaciones. Arrizabalaga y Wagman (1997: 36) expresan bien la esencia de este proceso: "No se trata de fijarnos como único objetivo la reducción del consumo, sino de profundizar en nuestra relación con las cosas para descubrir otras maneras de disfrutar una buena vida.» Otro aspecto en el que habría que hacer hincapié es la importancia que ostenta el fenómeno de la información, imprescindible para desenvolverse con un mínimo de éxito en el tejido social. Para lograr calidad es preciso estar informados, lo que exige controlar el flujo de datos, así como poseer criterios para seleccionar y gestionar un volumen de información que ya es abrumador.

Sin embargo - y aquí encontramos una tercera paradoja-, la calidad de vida es un auténtico elemento para el control social, basado en mecanismos que empujan a la acción, a la iniciativa y la innovación. En lo que se refería al consumo, había sido puesto de manifiesto por Bauman (1992: 99), quien, apoyándose en Foucault, señala cómo «la estimulación de una conducta funcionalmente indispensable para el sistema económico capitalista e inocua para el sistema político capitalista puede ahora dejarse en manos del mercado de consumo y sus atractivos. La reproducción del sistema capitalista se logra, por tanto,

18 Otras realidades novedosas e igualmente paradójicas que no es posible tratar aquí se refieren a la capacidad de la calidad de vida de reproducir el sistema capitalista, por cuanto se basa en mecanismos de producción y consumo, al mismo tiempo que posee la fuerza inicial para transformar beneficiosamente la optimización de procesos y la protección del medio ambiente. No menos paradójico es el hecho de cómo los estándares objetivos de calidad de vida contribuirían al proceso de homogeneización a escala planetaria, al mismo tiempo que los gustos personales y las satisfacciones individuales incrementan la diferenciación entre los miembros de cada sociedad. 
mediante la libertad individual y no a través de su supresión. En lugar de contabilizarse como coste fijo del sistema, toda la operación de "control social" puede ahora registrarse como activo del sistema»" ${ }^{19}$. Sin embargo, el control social a través del consumo se mueve en un plano externo al sujeto.

Lo que hace de la calidad de vida un elemento organizador de la vida de las personas más poderoso que el consumo no es su fundamento racional, como hemos visto. Su fuerza de contención, su capacidad de disciplina, tampoco procede de la limitación de los recursos o del proceso de aprendizaje, sino del propio carácter normativo de la calidad de vida. Según criterios externos, en primer lugar, porque se mide continuamente por patrones objetivos de calidad: medio ambiente, salud, nivel de vida material, etc., determinados por expertos socialmente reconocidos. Razones subjetivas son el hecho de que la calidad de vida se base en la necesidad de equilibrar el conjunto de satisfacciones que la generan y, sobre todo, la presencia de un proyecto personal que la rige en sus fines y sus actividades, que dota de sentido a todo el proceso.

Podría hablarse de un cambio en la situación del individuo en el mundo, tanto en la manera que el hombre tiene de verse a sí mismo como de estar realmente. Y esto, aplicable al entorno natural como al social ${ }^{20}$. Frente a la visión tradicional, de raíces clásicas, en la que cada ser humano es un actor que se ve obligado a interpretar uno o varios papeles sociales (Dahrendorf, 1973; Berger, 1988), encontraríamos otra comparación quizá más acorde con la presencia de la informática en los tiempos que corren. Sin desaparecer efectivamente el desempeño de los roles sociales, el ser humano estaría inmerso en una especie de gigantesco juego de ordenador, de videoaventura caracterizada por un tipo particular de estrategia, en la que el objetivo sería recorrer - y superar- una serie de "pantallas» o "niveles» de calidad de vida: en cada una se proponen nuevas habilidades y nuevas metas que hay que alcanzar: mientras se completan las fases del juego, se olvidan las penalidades y los logros anteriores, y se redefinen los objetivos que marcan las nuevas pautas de conducta, que orientan primordialmente a la acción. Dado que el agente está dotado de recursos, posee un gran margen de actuación, aunque siempre ha de ir buscando un camino, dentro de la amplia gama de opciones, aquel que se supone capaz de proporcionar la mayor calidad de vida. El juego es un continuo intercambio de recursos entre el agente y el sistema, ya que, como compensación por los beneficios que recibe, el jugador ha de estar continuamente realizando nuevas

19 El mismo Bauman compara la situación en que vive la mayoría de la gente en los países occidentales con la de quienes vivían en el bloque socialista y con la de quienes dependen de la asistencia social para vivir, expresando en qué medida es mucho mayor el control y, sobre todo, la humillación a los que están sometidos quienes se hallan en estas circunstancias.

${ }^{20}$ Las relaciones de los seres humanos con su entorno material están sufriendo la más llamativa transformación, por cuanto hemos pasado de tener que defendernos de la naturaleza a tener que protegerla de nosotros mismos. Temas como la contaminación en todas sus variedades, las fuentes de energía y su transmisión, la vida en el entorno creado de las ciudades, la salud ambiental, etc., forman parte indiscutible del área de la calidad de vida. 
apuestas. Recuperar el sentido de las acciones puede proporcionar un breve respiro, la pequeña pausa que sigue a cada nivel, a cada victoria, pero en seguida se verá inmerso en el logro de nuevas metas, activando sus fuerzas y gastando sus «vidas» en las actividades que ha de realizar. El juego carece de un final programado, porque no existe más final que la muerte física del jugador ${ }^{21}$. Y ni siquiera podríamos considerarlo como un final, sino más bien como la situación de alguien que abandona el juego, puesto que éste continúa para el resto.

Hage y Powers (1992) plantean estas transformaciones al mostrar que la sociedad postindustrial favorece el desarrollo de nuevos tipos humanos, caracterizados por poseer "yoes» creativos y personalidades innovadoras: adecuarse a las exigencias de la calidad de vida hace a las personas polivalentes, flexibles, dialogantes, con capacidad de iniciativa, etc. Es evidente que la solución a los problemas - grandes y pequeños - de la vida no podrá ya encontrarse en la que dieron generaciones anteriores: es preciso arriesgarse a encontrar nuevas fórmulas, que pasan por las tensiones que hemos descrito. Con palabras de Simmel, referidas precisamente a la aventura, podemos concluir que «la síntesis de las grandes categorías vitales [...] se consuma entre la actividad y la pasividad, entre lo que conquistamos y lo que nos es dado" (1988: 16).

\section{REFLEXIONES FINALES}

Como acertadamente indica Setién (1993: 402), «la calidad de vida no es un concepto neutro, ni la preocupación por la misma representa una idea libre de valores. Al contrario, el surgimiento del interés por la calidad de vida y el intento de reflejarlo en cualquier sociedad plantea un dilema social sobre lo que significa el desarrollo. Presenta un dilema porque lo que se discuten son objetivos sociales: dilema entre producir más suponiendo que con ello se garantiza el bienestar social o centrarse directamente en la mejora de la vida de los hombres». Con el surgimiento y difusión de los valores postmaterialistas, el debate señalado por Setién deja de estar exclusivamente en los despachos de las agencias decisorias para irrumpir en los hogares de las clases medias postindustriales, canalizándose en comportamientos de diversa índole, que afectarán indirectamente a los procesos de toma de decisiones.

Es preciso entender la búsqueda de la calidad de vida como motor de la actividad humana, como acción social encaminada a un fin, de no pequeñas repercusiones sociales. Si la idea de vida cotidiana ha supuesto alguna vez reproducción e inmovilidad, la búsqueda de la calidad por parte de los individuos en las sociedades industriales avanzadas obliga a replantearse estas cuestiones, por cuanto, legitimada socialmente, rige la actividad social e individual

${ }^{21}$ El contraste que existe entre los yuppies de los años ochenta y los downshifters de los noventa muestra otra paradójica realidad del juego de la calidad de vida: sólo se puede renunciar a él en aras de... una mayor calidad de vida 
y pone en juego numerosos recursos económicos, técnicos, culturales, sociales y políticos, introduciendo mayores dosis de complejidad social. De hecho, la calidad de vida es también una respuesta al hecho fundamental de la mundialización, puesta de manifiesto en su naturaleza global, reflejada en las acciones aparentemente más superficiales. Aunque suene prosaico - y, desde luego, paradójico-, la calidad de vida introduce la visión universal en el tipo de verdura que se come, en la marca de jabón que se elige, en el envase de plástico que se utiliza o se tira, en el viaje que se planifica: impregna lo cotidiano de un sentido trascendente hasta ahora insospechado, dotado de una fuerza increíble.

De esta manera, podemos pensar en los sujetos individuales como agentes de cambio social, ciertamente a pequeña escala, pero como hasta ahora no había tenido nunca lugar, habida cuenta de las circunstancias premodernas y modernas. No obstante, la calidad de vida no depende exclusivamente de los individuos, por lo que es una curiosa mezcla de acción y pasión, que conduce a un tipo de cambio social suave, de evolución lenta, sin rupturas globales, que al mismo tiempo impregna la vida corriente de pequeños traumas y continuos desajustes. Es una acción que no se rebela frente al statu quo, pero que tampoco parece dispuesta a su inmovilidad. Además, las personas encuentran en la calidad de vida un cauce para la participación ciudadana en creciente expansión: movimiento consumerista, asociacionismo, voluntariado social, etc. Esta tensa relación entre el agente y la sociedad forma parte de la dialéctica del cambio en el mundo actual, que convierte a los individuos en recursos inteligentes para su propia transformación. ¿Tiene la calidad de vida su lógica propia, más allá de la lógica del mercado o de la democracia? ¿Cómo será su influencia en el desarrollo de las políticas sociales y económicas? ¿Corresponde a una nueva situación en el "estatuto de ciudadanía» (Donati, 1993)? Hemos visto la presencia de mecanismos universalistas entre los habitantes de las sociedades avanzadas, pero ¿pueden llegar a identificarse mecanismos de reproducción propios de la calidad de vida, o caminará siempre a expensas de la economía? ¿Actuará la conciencia de la calidad de vida como un freno a las agresiones al medio ambiente, así como a la necesidad de dejar hueco y limitar el consumo, para favorecer el acceso a los países del Tercer Mundo?

Por otra parte $-\mathrm{y}$ de nuevo paradójicamente-, aunque se busca por sí misma, la calidad de vida sólo tiene un carácter instrumental: se orienta hacia el establecimiento de unas condiciones de vida favorables para el desarrollo de un proyecto personal y comunitario, pero no afirma nada sobre la naturaleza del mismo. Se debate entre los estándares sociales de calidad objetiva y el proyecto personal. Tiene un gran potencial de acción, por cuanto implica llevar a los demás esas mismas condiciones, pero no resuelve el problema del fin del ser humano, ni qué hay que hacer para lograr la felicidad. La calidad de vida sólo deja la puerta abierta: un proyecto de vida insuficiente puede realmente acabar haciendo de cada persona — en palabras de Baudrillard- un «hombre de colocación» poseedor de un proyecto que se «resigna» (1971: 228).

Lo indiscutible, en última instancia, es la consideración de la calidad de 
vida como medio de integración social de los seres humanos, no ya como miembros de una sociedad en particular, sino pertenecientes a la nueva "Sociedad», global y mundializada, que sigue siendo "un fenómeno dialéctico en cuanto que es un producto humano, y nada más que un producto humano, $y$, sin embargo, revierte continuamente sobre el propio causante. [...] Más aún, dentro de la sociedad, y como resultado de procesos vitales, el individuo se transforma en persona que alcanza y asume una identidad, y lleva a término los diversos proyectos que constituyen su vida» (Berger, 1981: 14). En opinión de Jameson (1996: 72), este nuevo sistema mundial ha roto nuestros esquemas cognitivos y cartográficos, al hacernos perder el sentido espacial y cultural. De ahí que «si alguna vez existe una forma política de la postmodernidad, su vocación será inventar y diseñar una cartografía cognitiva global, tanto a escala social como espacial». Ante ese reto nos encontramos.

\section{BIBLIOGRAFÍA}

Alonso, L. E., y CALlejo, J. (1994): «Consumo e individualismo metodológico: una perspectiva crítica», Política y Sociedad, n. ${ }^{\circ}$ 16, pp. 11-134.

ANDrÉs Orizo, F. (1992): «La sociedad del bienestar», en A. DE Miguel, La sociedad española 1992-93, Madrid, Alianza, pp. 225-244.

- (1996): Sistemas de valores en la España de los 90, Madrid, Centro de Investigaciones Sociológicas-Siglo XXI.

Arrizabalaga, A., y Wagman, D. (1997): Vivir mejor con menos. Con el testimonio de Fernando Savater, Madrid, Aguilar.

BAUDrILlard, J. (1971): El sistema de los objetos, Madrid, Siglo XXI.

- (1974): La sociedad de consumo, Barcelona, Plaza \& Janés.

BAUMAN, Z. (1992): Libertad, Madrid, Alianza.

BÉJAR, H. (1995): El ámbito intimo: Privacidad, individualismo y modernidad, Madrid, Alianza.

Berger, P. (1981): Para una teoría sociológica de la religión, Barcelona, Kairós.

- (1988): Introducción a la Sociología, México, Limusa.

BococK, R. (1995): El consumo, Madrid, Talasa.

Campbell, A.; Converse, Ph. E., y Rodgers, W. L. (1976): The Quality of American Life. Perceptions, Evaluations and Satisfactions, Nueva York, Russel Sage Foundation.

Campbell, C. (1995): "The Sociology of Consumption», en D. Miller (ed.): Acknowledging Consumption. A Review of New Studies, Londres, Routledge.

Camps, V. (1993): Paradojas del individualismo, Barcelona, Crítica.

Castillo Castillo, J. (1987): Sociedad de consumo a la española, Madrid, Eudema.

Centre D'Estudis Demografics (1988): La cohabitación en España: un estudio en Madrid y Barcelona, Madrid, Centro de Investigaciones Sociológicas.

Dahrendorf, R. (1973): Homo sociologicus, Madrid, Instituto de Estudios Políticos.

De Pablos, J. C.; Pascual, N., y GÓmeZ, Y. (1998): La búsqueda de la calidad de vida: una aproximación interpretativa, Granada, Facultad de CC.PP. y Sociología.

Del Campo, S. (Dir.) (1993): Tendencias sociales en España, Madrid, Fundación BBV.

Donati, P. (1993): La cittadinanza societaria, Roma-Bari, Laterza.

DURKHEIM, E. (1987): La división del trabajo social, Madrid, Akal.

Elias, N., y Dunning, E. (1992): Deporte y ocio en el proceso de civilización, México, FCE.

Featherstone, M. (1991): Consumer Culture and Post-modernism, Londres, Sage.

- (1995): Undoing culture. Globalization, Postmodernism and Identity, Londres, Sage. 
FuKuYAma, F. (1992): El fin de la historia y el último hombre, Barcelona, Planeta.

GALTUNG, J. (1972): «El problema de los indicadores sociales», en S. del Campo (Dir.), Los indicadores sociales a debate, Madrid, Euramérica.

Giddens, A. (1995): Modernidad e identidad del yo. El yo y la sociedad en la época contemporánea, Barcelona, Península.

GutiérRez Brito, J. (1995): «El consumo en la sociedad industrial avanzada», Estudios sobre Consumo, n. ${ }^{\circ} 34$, pp. 81-89.

Hage, J., y Powers, Ch. (1992): Post-industrial lives: roles and relationships in the 21st century, Newbury Park, Sage.

Handy, Ch. (1996): La edad de la paradoja, Barcelona, Apóstrofe.

IGLESIAS DE USSEL, J. (1994): «Familia», en M. Juárez (ed.), V Informe sociológico sobre la situación social en España, Madrid, Fundación Foessa, pp. 415-547.

INGLEHART, R. (1991): El cambio cultural en las sociedades industriales avanzadas, Madrid, CISSiglo XXI.

Jameson, F. (1996): Teoría de la postmodernidad, Madrid, Trotta.

KatOna, G. (1968): La sociedad de consumo de masas, Madrid, Rialp.

LUHMANN, N. (1995): "Individuo, individualidad, individualismo", Zona Abierta, n. ${ }^{\text {os }}$ 70-71, pp. 53-157.

MACKAY, H. (1997): Consumption and everyday life, Londres, Sage-Open University.

MAFFESOli, M. (1990): El tiempo de las tribus, Barcelona, Icaria.

- (1993): El conocimiento ordinario. Compendio de sociología, México, FCE.

Marina, J. A. (1995): Ética para náufragos, Barcelona, Anagrama.

MASLOW, A. (1975): Motivación y personalidad, Barcelona, Sagitario.

- (1982): La amplitud potencial de la naturaleza humana, México, Trillas.

Michalos, A. (1985): "Multiple Discrepancies Theory (MDT)», Social Indicators Research, vol. 16, n. ${ }^{4}$, pp. 347-413.

Miguel, A. de (1996): La sociedad española, 1995-1996, Madrid, Editorial Complutense.

Morin, E. (1995): Sociología, Madrid, Tecnos.

Nordenfelt, L. (1993): Quality of Life, Health and Happiness, Aldershot, Avebury

OCDE (1982): La liste OCDE des Indicateurs Sociaux, París, OCDE.

PÉREZ ADÁn, J. (1991): "Impacto de la revolución tecnológica en el trabajo humano", Revista de Trabajo y Seguridad Social, enero-marzo, pp. 85-108.

Pérez DíAz, V. (1993): La primacía de la sociedad civil: el proceso de formación de la España democrática, Madrid, Alianza.

Rodríguez, S. (1997): Creatividad en marketing directo, Bilbao, Deusto.

RoJeK, Ch. (1995): Decentring Leisure. Rethinking Leisure Theory, Londres, Sage.

Ruiz de Olabuénaga, J. I. (1994): «Ocio y estilos de vida», en M. Juárez (ed.), V Informe sociológico sobre la situación social en España, Madrid, Fundación Foessa, pp. 1880-2073.

- (Dir.) (1998): La juventud liberta: Género y estilos de vida en la juventud española, Madrid, Fundación BBV.

SAVATER, F. (1997): «El sentido común aplicado al consumo», en A. ArRizabalaga y D. WaGMAN, Vivir mejor con menos. Con el testimonio de Fernando Savater, Madrid, Aguilar, pp. 21 33.

SETIÉN, M. L. (1993): Indicadores sociales de calidad de vida. Un sistema de medición aplicado al País Vasco, Madrid, Centro de Investigaciones Sociológicas-Siglo XXI.

Simmel, G. (1988): Sobre la aventura, Barcelona, Península.

SQuicciarino, N. (1990): El vestido habla, Madrid, Cátedra.

SZTOMPKA, P. (1995): Sociología del cambio social, Madrid, Alianza.

Tocqueville, A. de (1989): La democracia en América, edición crítica de E. Nolla, 2 vols., Madrid, Aguilar. 


\begin{abstract}
This paper analyses the emergence of quality of life as one of the dominant values of postmaterialist societies: what engenders it in daily life, what is done to achieve it. Starting with classical distinctions in the concept of quality of life (objective elements and subjective satisfaction; equilibrium and wealth), the author goes on to examine other components in the habitual search for quality of life. This is achieved through consumption, but transcends it, providing an integrating vision which ranges from the elements of everyday life to one's own identity. Associated with the development of a fundamental project, quality of life implies a capacity for controlling the various facets of oneís life. In contrast, it involves the continuous bringing into play of available resources in a continuous succession of tensions and targets which the agent must resolve and meet in order to rediscover the meaning of his relationships with his social and natural surroundings. The author singles out certain paradoxes which impregnate quality of life with a strong dialectical content and illustrate the transformation of the relationship between the individual and society.
\end{abstract}

